\title{
A characterization of isochronous centres in terms of symmetries
}

\author{
Emilio Freire, Armengol Gasull and Antoni Guillamon
}

\begin{abstract}
We present a description of isochronous centres of planar vector fields $X$ by means of their groups of symmetries. More precisely, given a normalizer $U$ of $X$ (i.e., $[X, U]=\mu X$, where $\mu$ is a scalar function), we provide a necessary and sufficient isochronicity condition based on $\mu$. This criterion extends the result of Sabatini and Villarini that establishes the equivalence between isochronicity and the existence of commutators $([X, U]=0)$. We put also special emphasis on the mechanical aspects of isochronicity; this point of view forces a deeper insight into the potential and quadratic-like Hamiltonian systems. For these families we provide new ways to find isochronous centres, alternative to those already known from the literature.
\end{abstract}

\section{Introduction}

Along this paper we suppose to have a vector field $X$ on the plane with a centre at some critical point $p$. We are mainly concerned about the isochronicity problem, that is, to determine whether the periodic orbits around $p$ have the same period or not.

The pioneering works wondering about isochronicity were already given in the sixties by Levin and Shatz, Loud, Pleshkan and Urabe (see [7], [8], [11] and [14]). Nevertheless, until the beginning of this decade, the problem has not been deeply considered. The most relevant works are those of Sabatini and Villarini (see [13] and [15] and the references therein), where they settled the strong relationship between Lie brackets and isochronicity. In particular, they proved that $p$ is an isochronous centre if and only if there

2000 Mathematics Subject Classification: 34C14, 37C27, 17B80.

Keywords: isochronous centres, quadratic-like Hamiltonian systems, groups of symmetries, normalizers. 
exists a transversal commutator of this vector field. This idea has been used fruitfully by many authors and commutators for centres have been found for several families.

It is worth to say that the problem of isochronicity has also been thought of as a problem of linearization of centres, which is equivalent. This approach has been mainly used in the works of Mardešić, Moser-Jausslin, Rousseau and Toni (see [9] and [10]) and has opened as well a productive line to deal with hypothetical isochronous centres.

Our main result is in some sense a refinement of that introduced by Sabatini and Villarini. It will be proved in Section 2 and it states as follows:

Theorem 1 Consider a $\mathcal{C}^{1}$ vector field $X$ having a centre at a point $p$ and period annulus $\mathcal{P} \subset \mathbb{R}^{2}$. Let $U$ be a vector field $U \in \mathcal{C}^{1}(\mathcal{P})$, transversal to $X$ in $\mathcal{P} \backslash\{p\}$, and such that $[X, U]=\mu X$ for some $\mathcal{C}^{1}$ scalar function $\mu: \mathcal{P} \longrightarrow \mathbb{R}$. Let $\gamma=\{\mathbf{x}(t)\}$ be any periodic orbit of $X$ in $\mathcal{P}$ and denote by $T_{\gamma}$ its period.

Then, the centre is isochronous if and only if there is a neighbourhood of $p$ such that for any $\gamma$ contained in it,

$$
\int_{0}^{T_{\gamma}} \mu(\mathbf{x}(t)) d t=0
$$

Remark 2 The existence of $U$ and $\mu$ such that $[X, U]=\mu X$, is proved in [1] for general systems with a nondegenerate centre at $p$. The main difficulty for practical purposes is to find a way to compute $U$ and $\mu$. The vector field $U$ has a clear geometrical interpretation: since $X$ has a centre, it possesses a Lie group of symmetries; then, $U$ is the infinitesimal generator of this group. In Lie theory, the set of infinitesimal generators is called the normalizer of $X$, while the set of commuting vector fields is called the centralizer, see [16] for more details. In this context, Theorem 1 states that not only the centralizer but also a subset of the normalizer is connected to isochronicity. For short, an element of the normalizer of $X$ will be called a normalizer for $X$.

The above theorem can be used either to give a proof of the isochronicity for particular systems (see next examples) or to characterize the isochronous systems inside some families of centres. For instance, as we will see in Section 3, we are able to find a normalizer vector field for all Hamiltonian systems with Hamiltonian function

$$
H(x, y)=C(x) y^{2}+B(x) y+A(x)
$$

which are called quadratic-like Hamiltonian systems in [3]. 
Therefore, for (1.1) the characterization of the isochronous centres can be deduced from the integral condition given in Theorem 1, see Theorem 4. On the other hand, it seems not so easy to prove Theorem 4 either by applying Sabatini and Villarini's result or by finding a linearizing map, because one does not know, a priori, which systems inside this family are isochronous and as a consequence for which systems either a commutator vector field or a linearizing map can be found. Furthermore, even for concrete isochronous systems, it is not always an easy task to prove isochronicity by finding an explicit commutator, see for instance next Example 3.2 or Example 14, at the end of the paper. It is also difficult to find a linearizing map, see again Example 14. Finally, we notice that Theorem 1 also allows to prove some new characterizations of potential isochronous centres, see Corollary 5.

Examples 3 In the first example, both Theorem 1 and Sabatini and Villarini's method work. In the second example we illustrate the advantage of using Theorem 1 instead of looking for commutators.

3.1 In [4], the vector field

$X(x, y)=P(x, y) \frac{\partial}{\partial x}+Q(x, y) \frac{\partial}{\partial y}=y\left(-1+\frac{44}{9} x-\frac{16}{27} y^{2}\right) \frac{\partial}{\partial x}+\left(x+\frac{8}{9} y^{2}\right) \frac{\partial}{\partial y}$

is studied; a normalizer $U$ and a scalar function $\mu$ are found to be, respectively:

$$
\begin{aligned}
U(x, y) & =\frac{1}{27}\left(27 x-60 x^{2}-144 y^{2}+880 x y^{2}\right) \frac{\partial}{\partial x}+\frac{1}{9} y\left(9+40 x+80 y^{2}\right) \frac{\partial}{\partial y} \\
\mu(x, y) & =\frac{20}{3} Q(x, y) .
\end{aligned}
$$

In that reference, the authors construct a new normalizer $U-\frac{20 y}{3} X$ which is effectively a commutator of $X$. As a consequence of Sabatini and Villarini's result they get isochronicity. By using Theorem 1, the isochronicity follows just seeing that:

$$
\begin{aligned}
\int_{0}^{T} \mu(x(t), y(t)) d t & =\frac{20}{3} \int_{0}^{T} Q(x(t), y(t)) d t=\frac{20}{3} \int_{0}^{T} \frac{d y(t)}{d t} d t \\
& =\frac{20}{3}[y(T)-y(0)]=0,
\end{aligned}
$$

where $\gamma=\{(x(t), y(t)), 0 \leq t \leq T\}$ is a periodic orbit of $X$ of period $T$.

3.2 Consider the potential system with Hamiltonian $H(x, y)=y^{2} / 2+V(x)$, where $V(x)=x^{2}(x-2)^{2} /(x-1)^{2}$, that is,

$$
X(x, y)=-y \frac{\partial}{\partial x}+V^{\prime}(x) \frac{\partial}{\partial y},
$$


We will see how by using Theorem 1 we can give a proof of its isochronicity. On the other hand it seems not so easy to find a commutator vector field for it in order to apply Sabatini's and Villarini's result. Define, thus,

$$
U(x, y)=\frac{V(x)}{V^{\prime}(x)} \frac{\partial}{\partial x}+\frac{y}{2} \frac{\partial}{\partial y} \quad \text { and } \quad \mu(x, y)=\frac{x(x-2)}{\left(x^{2}-2 x+2\right)^{2}} .
$$

It is easy to check that $[X, U]=\mu X$. In Sections 3 and 4, see also Remark 7 and Example 8, it is explained a constructive way to get the above example and their associate normalizer. Let us apply Theorem 1. Denote by $\{(x(t), y(t))\}$ the time parameterization of a solution associated to the closed level curve $H(x, y)=h$ of $X$, and by $T_{h}$ its period. Denote by $x_{m}(h)$ (resp. $x_{M}(h)$ ) the negative (resp. positive) solution of the equation $V(x)=h$, for $h$ positive small enough. Then

$$
\int_{0}^{T_{h}} \mu(x(t)) d t=\sqrt{2} \int_{x_{m}(h)}^{x_{M}(h)} \frac{\mu(x)}{\sqrt{h-V(x)}} d x=(*),
$$

and by using the change of variables $u=g(x):=x(x-2) /(2(x-1))$, with inverse $x=g^{-1}(u)=1+u-\sqrt{1+u^{2}}$, we get

$$
\begin{aligned}
(*) & =\left.\sqrt{2} \int_{-\sqrt{h / 4}}^{\sqrt{h / 4}} \frac{\mu(x)}{\sqrt{h-V(x)}} \frac{8}{V^{\prime}(x)}\right|_{x=g^{-1}(u)} u d u \\
& =-\frac{\sqrt{2}}{2} \int_{-\sqrt{h / 4}}^{\sqrt{h / 4}} \frac{u}{\sqrt{h-4 u^{2}}\left(1+u^{2}\right)^{3 / 2}} d u=0 .
\end{aligned}
$$

Notice that the above change is constructed in order to have $V\left(g^{-1}(u)\right)=4 u^{2}$. This example has been already studied by using other methods in [14] and in [10, Eq. 7.17].

Finally, we use our results on quadratic-like Hamiltonian systems to give solutions to a classical mechanical problem: suppose that we have a particle moving in the $(z, x)$-plane on a $\mathcal{C}^{2}$ plane curve, $z=h(x)$, without friction, on which only the gravitational force acts, see Figure 1. Its Hamiltonian formulation, supposing that both the mass of the particle and the gravity acceleration constant are 1 , is

$$
H(x, y)=\frac{1}{2} \frac{1}{1+h^{\prime}(x)^{2}} y^{2}+h(x)
$$

At least three physical problems involving time are worth to be considered in this framework: 


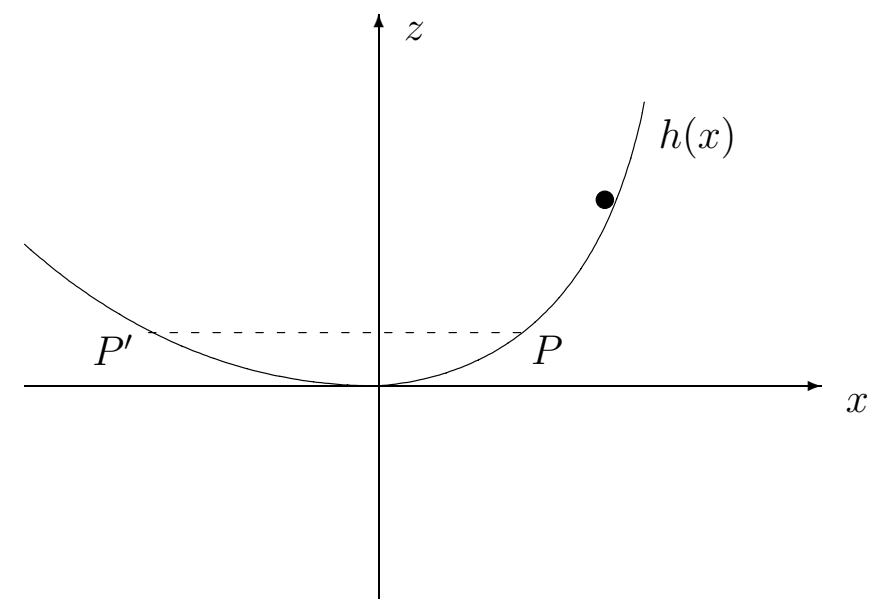

Figure 1: Ball moving on a plane curve.

- Braquistochronicity: Describing the shape of $h(x)$ that minimizes the time the particle takes from $P$ to the origin, whatever the point $P$ is. This problem was proposed in Acta eruditorum (1696) by Johann Bernouilli and solved by himself and many other famous mathematicians: Leibnitz, Newton, Jacob Bernouilli and de l'Hôpital. It was already considered by G. Galilei. It is well known that $z=h(x)$ is given by a family of cycloids.

- Tautochronicity: Describing the shape of $h(x)$ such that the time to go from $P$ to the origin does not depend on the point $P$. This problem was solved by Huygens in Horologium oscillatorium (1673). See Lemma 13 in Section 4. In this case the solution of this problem is also given by a family of cycloids. As far as we know, although braquistochronicity and tautochronicity problems have the same solutions there is not an easy explanation of this fact.

- Isochronicity: Describing the shape of $h(x)$ such that the time to go from $P$ to $P^{\prime}$ does not depend on the point $P$.

Of course, when $h(x)$ is symmetric, the last two problems coincide. The third problem was studied by Keller in [5]. Nevertheless, to our knowledge, no text gives explicit examples of the non-symmetric case which has a clear physical meaning. Here, we give one, see Example 14. 


\section{Proof of Theorem 1}

We assume by hypothesis that $[X, U]=\mu X$.

Suppose first that $X$ has an isochronous centre at $p$. By the result of Sabatini (see [13]), we know that there exists a vector field $Y$ transversal to $X$ in a punctured neighbourhood of $p$ and such that $[X, Y]=0$. Since $U$ and $X$ are also transversal in $\mathcal{P} \backslash\{p\}, Y$ can be expressed as $Y=f U+g X$ for some scalar functions $f$ and $g$. Then,

$$
[X, Y]=f[X, U]+X(f) U+X(g) X=(\mu f+X(g)) X+X(f) U=0 .
$$

Since $X$ and $U$ are transversal, both coefficients must vanish and $f, g$ and $\mu$ satisfy :

$$
\begin{aligned}
\mu f & =-X(g), \\
X(f) & =0 .
\end{aligned}
$$

From the second equation we get that $f$ is either a first integral of the vector field $X$ or a constant nonvanishing function. Set $\mathbf{x}=(x, y)$. Let $\gamma=\{\mathbf{x}(t)\}$ be a periodic orbit of $X$ of period $T_{\gamma}$ included in $\mathcal{P}$. Observe that from the first equation, using that $f$ is constant on $\gamma$,

$$
\begin{aligned}
0 & =\int_{0}^{T_{\gamma}}(f \mu)(\mathbf{x}(t)) d t+\int_{0}^{T_{\gamma}} X(g)(\mathbf{x}(t)) d t \\
& =f(\mathbf{x}(0)) \int_{0}^{T_{\gamma}} \mu(\mathbf{x}(t)) d t+\left.g(\mathbf{x}(t))\right|_{0} ^{T_{\gamma}}=f(\mathbf{x}(0)) \int_{0}^{T_{\gamma}} \mu(\mathbf{x}(t)) d t .
\end{aligned}
$$

If $f(\mathbf{x}(0))=0$ then $X$ would be parallel to $Y$ on $\gamma$. Hence,

$$
\int_{0}^{T_{\gamma}} \mu(\mathbf{x}(t)) d t=0
$$

for any $\gamma$ in a neighbourhood of $p$, as we wanted to prove.

Let us prove the converse. Consider a small neighbourhood of $p$ and assume that $\gamma$ is a $T_{\gamma}$-periodic orbit of $X$ contained in it. We claim that there is a ring-shaped neighbourhood $\mathcal{D}$ of $\gamma$, such that all the periodic orbits of $X$ have the same period $T_{\gamma}$. Clearly this fact will imply the isochronicity of $X$.

To prove the claim we will construct a commutator $Y$ of $X$ of the form $Y=U+g X$ on $\mathcal{D}$. Once $Y$ is constructed the claim will follow arguing as in [13].

Note that, by using (2.1), the existence of $Y$ is a consequence of the existence of a function $g$ such that $\mu=-X(g)$, defined in $\mathcal{D}$. In other 
words, $Y$ will exist if we can construct a solution $g(\mathbf{x})$ (global in all $\mathcal{D}$ ) of the first order quasi-linear partial differential equation

$$
g_{x}(\mathbf{x}) X_{1}(\mathbf{x})+g_{y}(\mathbf{x}) X_{2}(\mathbf{x})=-\mu(\mathbf{x}) .
$$

Consider the above partial differential equation with the initial condition

$$
\left.g(\mathbf{x})\right|_{\Sigma_{\varepsilon}}=0
$$

(any other initial condition will lead to a suitable $g$, but we choose the simplest one) where

$$
\Sigma_{\varepsilon}=\{\mathbf{u}(s),-\varepsilon \leq s \leq \varepsilon\},
$$

being $q$ a point of $\gamma$ and $\mathbf{u}(s)$ the solution associated to the vector field $U$, which passes through $q$ when $s=0$.

By using the characteristic method, the surface solution of (2.3) in the $(\mathbf{x}, g)$-coordinates, satisfying (2.4) is given by the parametric expression

$$
\left\{\begin{array}{l}
\mathbf{x}=\alpha(t, s):=\varphi(t, \mathbf{u}(s)), \\
g=\beta(t, s):=-\int_{0}^{t} \mu(\varphi(w, \mathbf{u}(s))) d w
\end{array}\right.
$$

being $\varphi\left(t, \mathbf{x}_{0}\right)$ the solution of the Cauchy problem

$$
\left\{\begin{aligned}
d \mathbf{x}(t) / d t & =X(\mathbf{x}(t)) \\
\mathbf{x}(0) & =\mathbf{x}_{0}
\end{aligned}\right.
$$

The transversality of $X$ and $U$ on $\Sigma_{\varepsilon}$ implies, by using the Implicit Function theorem, that the surface $(\mathbf{x}, g)=(\alpha(t, s), \beta(t, s))$ for $|s| \leq \varepsilon$ and $\varepsilon$ and $t$ small enough can be expressed as $g=g(\mathbf{x})$ for some smooth $g$. To define globally $g$ on some ring $\mathcal{D}$, containing $\gamma$, consider

$$
\mathcal{D}:=\bigcup_{|s| \leq \varepsilon}\{\varphi(t, \mathbf{u}(s)), t \in \mathbb{R}\} \quad \text { and } \quad \mathcal{R}:=\left\{(t, s):|s| \leq \varepsilon, t \in \mathbb{R} /\left[0, T_{s}\right]\right\} .
$$

The fact that fixed any $s \in(-\varepsilon, \varepsilon)$, the orbit $\varphi(t, \mathbf{u}(s))$ is $T_{s}$-periodic, implies that the map $\alpha: \mathcal{R} \rightarrow \mathcal{D}$ is smooth and bijective. On the other hand, the map $\beta$ is well defined in $\mathcal{R}$ because, by hypothesis,

$$
\int_{0}^{T_{s}} \mu(\varphi(w, \mathbf{u}(s))) d w=0
$$

Therefore $g=g(\mathbf{x}):=\beta\left(\alpha^{-1}(\mathbf{x})\right)$ is a solution of (2.3) defined in $\mathcal{D}$, and the claim follows. 


\section{Isochronicity in quadratic-like Hamiltonians.}

We will now face to the applications of Theorem 1 to the problem of isochronicity in quadratic-like Hamiltonian systems. That is, we consider $H(x, y)=$ $A(x)+B(x) y+C(x) y^{2}$, satisfying $C(0) \neq 0$.

By the symplectic "translation", $(x, y) \rightarrow(x, y+B(x) /(2 C(x)))$, one can suppose that $B(x) \equiv 0$. That is, if we define

$$
G(x):=\frac{4 A(x) C(x)-B(x)^{2}}{4 C(x)},
$$

then we can write the transformed Hamiltonian as

$$
H(x, y)=G(x)+C(x) y^{2} .
$$

At this point we could go further and apply the new symplectic change,

$$
(x, y) \rightarrow\left(\int_{0}^{x} \frac{d u}{\sqrt{2 C(u)}}, y \sqrt{2 C(x)}\right),
$$

to transform $H$ into a potential Hamiltonian, that is, of the form $H(x, y)=$ $y^{2} / 2+V(x)$. However, if we keep using (3.2), we will be able to obtain characterizations of isochronous centres more directly related to the functions with physical meaning. So, from now on, we will consider as $X$ the Hamiltonian vector field associated to (3.2), that is:

$$
X=\left\{\begin{array}{l}
\dot{x}=-2 y C(x), \\
\dot{y}=G^{\prime}(x)+C^{\prime}(x) y^{2},
\end{array}\right.
$$

Without loss of generality we assume that the critical point $p$ is at $(0,0)$. The conditions that imply that the origin of (3.3) is a nondegenerate centre read as:

Hypothesis $\mathbf{H}$. There exists an interval $I=\left[x_{L}, x_{R}\right] \subset \overline{\mathbb{R}}$, with $x_{L}<0<x_{R}$ where $C$ and $G$ are $\mathcal{C}^{2}$ functions, $C$ is always positive and $\mathrm{G}$ satisfies: $G(0)=$ $G^{\prime}(0)=0, G^{\prime \prime}(0)>0, G\left(x_{L}\right)=G\left(x_{R}\right)$, and $x G^{\prime}(x)>0$ for all $x \in I \backslash\{0\}$.

We are interested in the level curves of the Hamiltonian (3.2) ranging from $h=0$ to $h=h_{\max }=H\left(0, x_{L}\right)=G\left(x_{L}\right)=H\left(0, x_{R}\right)=G\left(x_{R}\right)$.

Our purpose is to apply Theorem 1 to (3.3) and, at the same time, obtain specific methods to check or characterize isochronicity for this family of systems. We have obtained two characterizations based on the knowledge of the function $\mu$ and the use of suitable variables. To state our result it is convenient to introduce some auxiliary functions

$$
g(x):=x \sqrt{\frac{2 G(x)}{G^{\prime \prime}(0) x^{2}}}, \quad F(x):=\int_{0}^{x} \frac{d \xi}{\sqrt{C(\xi)}} .
$$


Notice that if Hypothesis $\mathrm{H}$ is satisfied, then $g(x)$ is invertible in a neighbourhood of 0 . Finally we introduce the function $q(u)$, by the equality

$$
F\left(g^{-1}(u)\right)=\alpha^{*} u-q(u)
$$

where $\alpha^{*}$ is the only real constant such that $q(u)$ starts with second order terms in a neighbourhood of 0 , i. e. such that $q(0)=q^{\prime}(0)=0$.

Theorem 4 Consider the Hamiltonian vector field (3.3),

$$
X=\left\{\begin{array}{l}
\dot{x}=-2 y C(x), \\
\dot{y}=G^{\prime}(x)+C^{\prime}(x) y^{2},
\end{array}\right.
$$

and suppose that the Hypothesis $H$ is fulfilled. Then the following conditions are equivalent:

1. The centre at the origin is isochronous.

2. $\frac{F}{2}-F^{\prime} \frac{G}{G^{\prime}}$ is either 0 or a function of $G$ which vanishes at 0 (recall that $F$ is given in (3.4)).

3. The function $q$ introduced in (3.5) is either 0 or an even function.

Proof of Theorem 4. Consider the vector field $X$ given in (3.3). First of all, let us notice that defining the new vector field

$$
U=\left\{\begin{aligned}
\dot{x} & =\frac{G(x)}{G^{\prime}(x)} \\
\dot{y} & =\left(1-\frac{G(x)}{G^{\prime}(x)} \frac{C^{\prime}(x)}{C(x)}\right) \frac{y}{2},
\end{aligned}\right.
$$

and the function

$$
\mu(x)=-\frac{1}{2}+\left(\left(\frac{G(x)}{G^{\prime}(x)}\right)^{\prime}-\frac{G(x)}{2 G^{\prime}(x)} \frac{C^{\prime}(x)}{C(x)}\right) .
$$

we have that $[X, U]=\mu X$ in some neighbourhood of the origin. We will prove that $2 \Rightarrow 1 \Rightarrow 3 \Rightarrow 2$.

$(2 \Rightarrow \mathbf{1})$ : Assume that $\frac{F}{2}-F^{\prime} \frac{G}{G^{\prime}}=\varphi(G)$, for some function $\varphi: \mathbb{R} \rightarrow \mathbb{R}$ vanishing at 0 . Fix an energy level $h=C(x) y^{2}+G(x)$. Consider the corresponding level curve $\gamma_{h}$ and call $T$ its period. Define as $x_{m}$ and $x_{M}$ the two intersections of $\gamma_{h}$ with the $x$-axis. Using the symmetry on $y$ in (3.3), we have that

$$
\int_{0}^{T} \mu(\mathbf{x}(t)) d t=\int_{x_{m}}^{x_{M}} \frac{\mu(x) d x}{\sqrt{C(x)} \sqrt{h-G(x)}} .
$$


According to (3.7), the hypothesis and the fact that

$$
\frac{\mu(x)}{\sqrt{C(x)}}=-\left(\frac{F(x)}{2}-F^{\prime}(x) \frac{G(x)}{G^{\prime}(x)}\right)^{\prime}
$$

it follows that

$$
\begin{aligned}
\int_{x_{m}}^{x_{M}} \frac{\mu(x) d x}{\sqrt{C(x)} \sqrt{h-G(x)}} & =-\int_{x_{m}}^{x_{M}} \frac{d}{d x} \varphi(G(x)) \frac{d x}{\sqrt{h-G(x)}} \\
& =-\int_{x_{m}}^{x_{M}} \varphi^{\prime}(G(x)) G^{\prime}(x) \frac{d x}{\sqrt{h-G(x)}} .
\end{aligned}
$$

Since $G(x)$ is a parabola-like function and ranges from 0 to $h$ when $H=h$, we can split the last integral in two parts and apply in each of them the change $z=G(x)$. At the end, we obtain that

$$
\begin{aligned}
& \int_{0}^{T} \mu(x(t), y(t)) d t= \\
& \quad=-\int_{x_{m}}^{0} \varphi^{\prime}(G(x)) G^{\prime}(x) \frac{d x}{\sqrt{h-G(x)}}-\int_{0}^{x_{M}} \varphi^{\prime}(G(x)) G^{\prime}(x) \frac{d x}{\sqrt{h-G(x)}} \\
& \quad=-\int_{h}^{0} \varphi^{\prime}(z) \frac{d z}{\sqrt{h-z}}-\int_{0}^{h} \varphi^{\prime}(z) \frac{d z}{\sqrt{h-z}}=0,
\end{aligned}
$$

and so, by Theorem 1 , the origin is an isochronous centre.

$(\mathbf{1} \Rightarrow \mathbf{3})$ : We directly apply Theorem 1 by computing $\mu$ in terms of $u=g(x)$. Straightforward computations from (3.7) give:

$$
\mu(u)=\frac{-u q^{\prime \prime}(u)}{2\left(\alpha^{*}-q^{\prime}(u)\right)} .
$$

We impose that the integral of $\mu$ must vanish. Then, using again (3.8) and the definition of $F$ we obtain that

$$
0=\int_{0}^{T} \mu(x(t)) d t=\int_{x_{m}}^{x_{M}} \frac{\mu(x) d x}{\sqrt{C(x)} \sqrt{h-G(x)}}=\int_{x_{m}}^{x_{M}} \frac{\mu(x) F^{\prime}(x) d x}{\sqrt{h-G(x)}} .
$$

Changing to the variable $u=g(x)$ we have that

$$
\frac{1}{2} \int_{-\sqrt{h / \alpha}}^{\sqrt{h / \alpha}} \frac{u q^{\prime \prime}(u) d u}{\sqrt{h-\alpha u^{2}}}=\frac{1}{2} \int_{0}^{\sqrt{h / \alpha}} \frac{u\left(q^{\prime \prime}(u)-q^{\prime \prime}(-u)\right) d u}{\sqrt{h-\alpha u^{2}}}=0 .
$$

Now, applying the change $u=\sqrt{h / \alpha} \cos \theta$, we get

$$
\frac{\sqrt{h}}{\alpha} \int_{0}^{\pi / 2} \cos \theta\left(q^{\prime \prime}(\sqrt{h / \alpha} \cos \theta)-q^{\prime \prime}(-\sqrt{h / \alpha} \cos \theta)\right) d \theta=0,
$$


for all $h \in\left(0, h_{\max }\right]$. Following the Lemma in [7] we get that $q^{\prime \prime}(u)=q^{\prime \prime}(-u)$ whenever $u \in\left(0, \sqrt{h_{\max } / \alpha}\right]$. Then, $q^{\prime \prime}$ is an even function and so $q$ is. Hence statement 3 holds.

(3 $\Rightarrow 2)$ : Firstly note that by introducing the variable $u=g(x)$ we have that $G(x)=\alpha u^{2}$ for some $\alpha>0$ and that $x=g^{-1}(u):=u-p(u)$ for some function $p$ satisfying $p(0)=p^{\prime}(0)=0$. Remember also that $F(x)=$ $F\left(g^{-1}(u)\right)=\alpha^{*} u-q(u)$ with $q(0)=q^{\prime}(0)=0$ and suppose that $q(u)$ is an even function. It is clear that

$$
\begin{aligned}
\frac{F(x)}{2}-\left.F^{\prime}(x) \frac{G(x)}{G^{\prime}(x)}\right|_{x=g^{-1}(u)} & =\frac{\alpha^{*} u-q(u)}{2}-\frac{\left(\alpha^{*}-q^{\prime}(u)\right)}{\left(g^{-1}\right)^{\prime}(u)} \frac{u^{2}\left(g^{-1}\right)^{\prime}(u)}{2 u} \\
& =\frac{1}{2}\left(u q^{\prime}(u)-q(u)\right) .
\end{aligned}
$$

Notice that $u q^{\prime}(u)-q(u)$, is an even function of $u$ that vanishes at 0 . This means that it is either 0 or that it can be written as a function of $u^{2}$ and so as a function of $G$ as statement 2 requires.

As a consequence of Theorem 4, in next corollary we give two new characterizations of the isochronous systems for the so called potential systems

$$
\left\{\begin{array}{l}
\dot{x}=-y \\
\dot{y}=v(x)
\end{array}\right.
$$

coming from $H(x, y)=\frac{1}{2} y^{2}+V(x)$, where $V$ a $\mathcal{C}^{2}$ function such that $V(0)=$ $V^{\prime}(0)=0$ and $V^{\prime \prime}(0) \stackrel{2}{=} \alpha>0$ and $v(x)=V^{\prime}(x)$. Note that potential systems correspond to our case $G(x) \equiv A(x) \equiv V(x), B(x) \equiv 0, C(x) \equiv 1 / 2$ and $F(x)=\sqrt{2} x$.

Corollary 5 Consider the vector field (3.9). Then the following statements are equivalent:

1. The centre at the origin is isochronous.

2. $\frac{x}{2}-\frac{V}{V^{\prime}}$ is either 0 or a function of $V$ that vanishes at the origin.

3. The function $u-g^{-1}(u)$ is either 0 or an even function, where $g^{-1}$ is the inverse of the map

$$
g(x):=x \sqrt{\frac{2 V(x)}{V^{\prime \prime}(0) x^{2}}} .
$$


Remark 6 A first characterization of potential isochronous centres was given by Urabe in [14], see next condition 4. Other two equivalent conditions were given in [3]. See also [6] and [17]. We include these other equivalent statements here for sake of completeness:

$$
\text { 4. }\left.\quad \frac{d}{d x} V(x)\right|_{x=g^{-1}(u)}=v\left(g^{-1}(u)\right)=2 \alpha \frac{u}{1+S(u)},
$$

where $S(u)$ is an arbitrary smooth odd function.

5 .

$$
V_{+}^{-1}(h)-V_{-}^{-1}(h)=\frac{2}{\sqrt{\alpha}} \sqrt{h},
$$

where $V_{+}^{-1}(h)$ (resp. $\left.V_{-}^{-1}(h)\right)$ is the positive (resp. negative) solution of $V(x)=h$ for $h$ in a neighbourhood of 0 .

6. There exists a strict involution $\sigma$ such that

$$
V(x)=\frac{\alpha}{4}(x-\sigma(x))^{2}
$$

for all $x$ in a neighbourhood of 0 .

\section{Examples}

Firstly we will give some examples of potential isochronous systems. They will be constructed from the following consequence of Corollary 5 .

Remark 7 Following 3 of Corollary 5, if the origin of a potential system is isochronous then the function $u-g^{-1}(u)$ has to be either 0 or an even function in the variable $u$, say $\phi\left(u^{2}\right)$. If we introduce the change of variables $u=g(x)$, it is satisfied that $V(x)=\alpha u^{2}$. Therefore the parametric expression of a function $V=V(x)$ associated to any potential isochronous centre is

$$
x=u-\phi\left(u^{2}\right), \quad V=\alpha u^{2},
$$

for some $\alpha>0$ and some function $\phi$ vanishing at zero. Notice also that an implicit expression for such $a V$ is

$$
V=\alpha(x+\hat{\phi}(V))^{2}
$$

where $\hat{\phi}(V)=\phi(V / \alpha)$. 
Examples 8 The following choices of $\phi$ or $\hat{\phi}$ in the above remark produce different examples of potential isochronous centres:

8.1 The function $\phi(z)=\sqrt{1+z}-1$ gives

$$
V(x)=\alpha \frac{x^{2}(x-2)^{2}}{(x-1)^{2}},
$$

for some $\alpha>0$, which already appears in [14] and in formula 7.17 of [10].

8.2 The function $\hat{\phi}(z)=\beta z^{m}, m \in \mathbb{N}$ gives that $V$ is a branch of the algebraic curve

$$
V=\alpha\left(x+\beta V^{m}\right)^{2} .
$$

For $m=1$, the $V$ obtained gives the example of isochronous system given in formula D.1 of [12].

8.3 The function $\hat{\phi}(z)=z /(1+z)$ gives that $V$ is a branch of the algebraic curve

$$
(1+V)^{2} V=\alpha(x(1+V)+V)^{2} .
$$

Note that Examples 8.1 and 8.2 define an isochronous centre just in a neighbourhood of the origin, while Example 8.3 defines a global isochronous centre because the change $x=g^{-1}(u)$ is in this case a global one.

For quadratic-like Hamiltonian systems, we explore as well some ways to create isochronous centres.

Corollary 9 Let $V$ be a function such that the potential system associated to $H(x, y)=y^{2} / 2+V(x)$ has an isochronous centre at the origin. Then the following quadratic-like Hamiltonian systems (3.3) have also an isochronous centre at the origin.

1. Systems satisfying that $G(x)=V(x)$ and

$$
C(x)=\left(\alpha^{*}+\frac{d}{d x} \Phi(V(x))\right)^{-2},
$$

where $\alpha^{*}>0$ and $\Phi$ is a smooth function which vanishes at the origin.

2. Systems satisfying that $G(x)=V(x)$ and

$$
C(x)=V(x)\left(\beta V^{\prime}(x)-\delta \sqrt{V(x)}\right)^{-2},
$$

where $\beta>0$ and $2 \beta \sqrt{\alpha}>\delta$. Remember that $\alpha>0$ is given by the relation $V\left(g^{-1}(u)\right)=\alpha u^{2}$. 
Proof: (1) Since the potential system has an isochronous centre at the origin, taking $G(x)=V(x)$ we know, from 3 of Corollary 5 that $x=g^{-1}(u)=$ $u-p(u)$, with $p$ an even function. Remember that $V\left(g^{-1}(u)\right)=\alpha u^{2}$. On the other hand

$$
F(x)=\int_{0}^{x} \frac{d z}{\sqrt{C(z)}}=\int_{0}^{x}\left(\alpha^{*}+\frac{d}{d z} \Phi(V(z))\right) d z=\alpha^{*} x+\Phi(V(x)) .
$$

Therefore

$$
F\left(g^{-1}(u)\right)=\alpha^{*}(u-p(u))+\Phi\left(\alpha u^{2}\right):=\alpha^{*} u-q(u) .
$$

So, $q(u)=\alpha^{*} p(u)-\Phi\left(\alpha u^{2}\right)$ is also an even function. By using 3 of Theorem 4 we obtain that the centre is isochronous, as we wanted to prove.

(2) Arguing as in case (1) we obtain that

$$
F(x)=\int_{0}^{x}\left(\beta \frac{V^{\prime}(z)}{\sqrt{V(z)}}-\delta\right) d z=2 \beta \sqrt{V(x)}-\delta x .
$$

Therefore

$$
F\left(g^{-1}(u)\right)=(2 \beta \sqrt{\alpha}-\delta) u+\delta p(u)
$$

with $p(u)$ even. Again, by using 3 of Theorem 4 the result follows.

\section{Examples 10}

10.1 Take $G(x)=(x(x-2) /(x-1))^{2}$. By Example 8.1 we have that the function $V=G$ has associated an isochronous potential system. By using part 1 of Corollary 9 with $\Phi(x) \equiv x$ and $\alpha^{*}=8$, or part 2 of Corollary 9 we obtain the quadratic-like isochronous centres given by $C(x)=$

$$
\frac{(x-1)^{6}}{4\left(x^{4}-6 x^{2}+8 x-4\right)^{2}},
$$

or

$$
\frac{(x-1)^{4}}{\left((\delta-2 \beta) x^{2}-2(\delta-2 \beta) x+\delta-4 \beta\right)^{2}}, \quad \text { with } \quad \delta<4 \beta,
$$

respectively.

10.2 Take $G(x)=x^{2} / 2$. By using part 1 of Corollary 9 again, with an arbitrary function $\Phi(x)$ and an arbitrary $\alpha^{*}>0$ we get that the quadratic-like systems with $C(x)=\left(\alpha^{*}+\Psi(x)\right)^{-2}$, being $\Psi(x)$ an arbitrary odd function, have also an isochronous centre at the origin. 
Corollary 11 Take an arbitrary smooth function $C(x)$ such that $C(0)>0$, and an arbitrary positive constant $\beta$. Then the quadratic-like Hamiltonian systems satisfying

$$
G(x)=\beta(F(x))^{2}=\beta\left(\int_{0}^{x} \frac{d z}{\sqrt{C(z)}}\right)^{2}
$$

have an isochronous centre at the origin.

Proof: Notice that the condition $\frac{F}{2}-F^{\prime} \frac{G}{G^{\prime}}=0$ is equivalent to $G(x) \equiv$ $\beta(F(x))^{2}$, for some $\beta \in \mathbb{R}$. Therefore from part 2 of Theorem 4 the result follows.

Remark 12 (i) Observe that when $\frac{F}{2}-F^{\prime} \frac{G}{G^{\prime}}=0$ then $\mu=0$ and (3.6) is a commutator of the quadratic-like Hamiltonian system.

(ii) Note also that the relation $G=\beta F^{2}$ given in Corollary 11 (which corresponds to $\left.\frac{F}{2}-F^{\prime} \frac{G}{G^{\prime}}=0\right)$ is equivalent to the condition of isochronicity, $C(x) G^{\prime}(x)^{2}=4 \beta G(x)$, given in [3] for quadratic-like Hamiltonian systems. This condition turns out to be necessary and sufficient when both $G$ and $C$ are even. We can also prove that this condition becomes necessary in the following way: using part 2 of Theorem 4 we have that there exists some $\varphi$ such that $\frac{F}{2}-F^{\prime} \frac{G}{G^{\prime}}=\varphi(G)$.

The evenness of $G$ and $C$ implies the oddness of $F$ and $\frac{F}{2}-F^{\prime} \frac{G}{G^{\prime}}$. So, we have that $G$ is even and for some $\varphi, \varphi(G)$ is odd. This can only happen if $\varphi=0$.

As we will see in the next lemma, the above condition particularized to the Hamiltonian system (1.2), gives the classical solution to the tautochronicity problem. We include it for the sake of completeness.

Lemma 13 Consider the quadratic-like Hamiltonian system (1.2),

$$
H(x, y)=\frac{1}{2} \frac{1}{1+h^{\prime}(x)^{2}} y^{2}+h(x)
$$

and assume that $h(x)$ is an even function vanishing at 0 . Then the only solutions of the isochronicity and tautochronicity problems associated to it, are given by the following family of cycloids

$$
x=k(w+\sin w), \quad h=k(1-\cos w),
$$

where $k>0$, and $w$ is a parameter which ranges between $-\pi$ and $\pi$. 
Proof: If $h$ is even, then

$$
C(x)=\frac{1}{2} \frac{1}{1+h^{\prime}(x)^{2}}
$$

is also even and by (ii) of Remark 12 we have that $h(x)=\beta F(x)^{2}$ for some positive $\beta$ is an equivalent condition to isochronicity. This last equation writes as

$$
\sqrt{h(x)}=\sqrt{\beta} \int_{0}^{x} \sqrt{2\left(1+h^{\prime}(z)^{2}\right)} d z
$$

or equivalently as the following implicit ordinary differential equation

$$
h(x)\left(1+\left(h^{\prime}(x)\right)^{-2}\right)=2 k,
$$

for some positive $k$. By introducing a new variable $w$ such that $h^{\prime}(x)=$ $\tan (w / 2)$, we obtain that

$$
x=k(w+\sin w), \quad h=k(1-\cos w),
$$

which is the parametric expression of a family of cycloids.

On the other hand, in the mechanical approach of the introduction we have put special emphasis in the problem of isochronicity in front of that of tautochronicity. In particular, breaking the symmetry we can achieve solutions different from cycloids, as next example shows.

Example 14 Consider that

$$
C(x)=\frac{1}{2\left(1+h^{\prime}(x)^{2}\right)}
$$

and take $h$ such that $h\left(g^{-1}(u)\right)=u^{2}$ and $F\left(g^{-1}(u)\right)=\alpha^{*} u-\frac{k}{2} u^{2}$, for $\alpha^{*}$ and $k$ positive. We then develop both $F^{\prime}(x)$ and $C(x)$ in terms of the variable $u=g(x)$ :

$$
\begin{aligned}
\frac{d F}{d x} & =\frac{d F}{d u} \frac{1}{1-p^{\prime}(u)}=\frac{\alpha^{*}-k u}{1-p^{\prime}(u)} \\
C\left(g^{-1}(u)\right) & =\frac{1}{2} \frac{1}{1+\left(\frac{d h}{d u} \frac{1}{1-p^{\prime}(u)}\right)^{2}}=\frac{1}{2} \frac{\left(1-p^{\prime}(u)\right)^{2}}{\left(1-p^{\prime}(u)\right)^{2}+4 u^{2}} .
\end{aligned}
$$

Then, imposing that $F^{\prime}(x)^{2}=\frac{1}{C(x)}$, we obtain the equality:

$$
\frac{\left(\alpha^{*}-k u\right)^{2}}{\left(1-p^{\prime}(u)\right)^{2}}=\frac{2\left(\left(1-p^{\prime}(u)\right)^{2}+4 u^{2}\right)}{\left(1-p^{\prime}(u)\right)^{2}}
$$


If we take now $k=2 \sqrt{2}$ and $\alpha^{*}=\sqrt{2}$ (other values of $k$ would lead to logarithmic functions and cumbersome expressions), we get that

$$
p(u)=u+\frac{1}{6}(1-4 u)^{3 / 2}-\frac{1}{6}, \quad \text { for } u<\frac{1}{4}
$$

and thus, from the relation $x=u-p(u)$,

$$
u=\frac{1}{4}\left(1-(1-6 x)^{2 / 3}\right), \quad \text { for } x<\frac{1}{6} .
$$

Finally, we obtain:

$$
h(x)=\frac{1}{16}\left(1-(1-6 x)^{2 / 3}\right)^{2} .
$$

The curve obtained $z=h(x)$ is clearly non-symmetric and so, it is an example of isochronicity but not of tautochronicity.

\section{References}

[1] Algaba, A., Freire, E., Gamero, E.: Isochronicity via normal form. Qual. Theory Dyn. Syst. 1 (2000), 133-156.

[2] Chavarriga, J., García, I., Giné, J.: On Lie's symmetries for planar polynomial differential systems. Nonlinearity 14 (2001), 863-880.

[3] Cima, A., Mañosas, F., Villadelprat, J.: Isochronicity for several classes of Hamiltonian systems. J. Differential Equations 157 (1999), no. 2, 373-413.

[4] García, I.: Contribution to the qualitative study of planar vector fields. Ph. D. Thesis, Universitat Autònoma de Barcelona, 2000.

[5] Keller, J. B.: Determination of a potential from its energy levels and undetectability of quantization at high energy. Amer. J. Phys. 30 (1962) $22-26$.

[6] Keller, J. B.: Inverse problems. Amer. Math. Monthly 83 (1976), no. 2, $107-118$.

[7] Levin, J. J., Shatz, S. S.: Nonlinear oscillations of fixed period. J. Math. Anal. Appl. 7 (1963), 284-288.

[8] Loud, W.S.: Behavior of the period of solutions of certain plane autonomous systems near centers. Contributions to Differential Equations 3 (1964), 21-36.

[9] Mardešić, P., Moser-Jauslin, L., Rousseau, C.: Darboux linearization and isochronous centers with a rational first integral. J. Differential Equations 134 (1997), no. 2, 216-268. 
[10] Mardešić, P., Rousseau, C., Toni, B.: Linearization of isochronous centers. J. Differential Equations 121 (1995), no. 1, 67-108.

[11] Pleshran, I. I.: A new method of investigating the isochronicity of a system of two differential equations. J. Differential Equations 5 (1969), 796-802.

[12] Rothe, F.: Remarks on periods of planar Hamiltonian systems. SIAM J. Math. Anal. 24 (1993), 129-154.

[13] Sabatini, M.: Characterizing isochronous centres by Lie brackets, Differential Equations Dynam. Systems 5 (1997), 91-99.

[14] Urabe, M.: Potential forces which yield periodic motions of a fixed period. J. Math. Mech. 10 (1961), 569-578.

[15] Villarini, M.: Regularity properties of the period function near a centre of a planar vector field. Nonlinear Anal. 19 (1992), 787-803.

[16] Walcher, S.: Multi-parameter symmetries of first order ordinary differential equations. J. Lie Theory 9 (1999), 249-269.

[17] Zampieri, G.: On the Periodic Oscillations of $\ddot{x}=g(x)$. J. Differential Equations 78 (1989), 74-88.

Recibido: 18 de abril de 2002

Emilio Freire

E. S. Ingenieros

Universidad de Sevilla

Camino de los Descubrimientos s.n.

41092 Sevilla, Spain

emilio@matinc.us.es

Armengol Gasull

Dept. de Matemàtiques, Edifici Cc

Universitat Autònoma de Barcelona

08193 Bellaterra, Barcelona, Spain

gasull@mat.uab.es

Antoni Guillamon

Dept. de Matemàtica Aplicada I

Universitat Politècnica de Catalunya

Dr. Marañón n. 44-50

08028, Barcelona

antoni.guillamon@upc.es

The first author is partially supported by the DGICYT grant no. PB98-1152 and Junta de Andalucia grant no. TIC-0130. The other authors are partially supported by the DGES grant no. BFM2002-04236-C02-2 and CONACIT grant no. 2001SGR00173. 